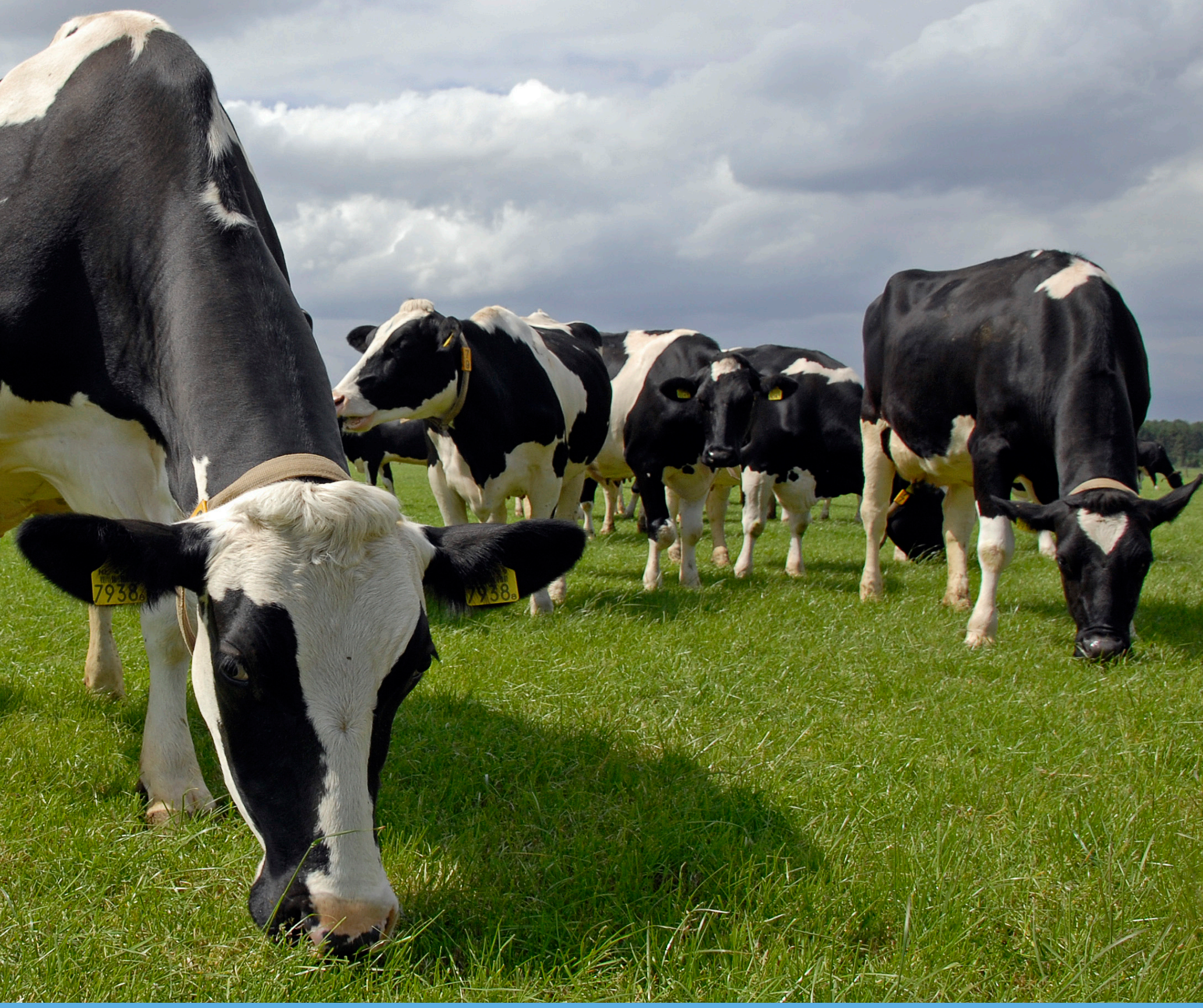

Rassenbericht Grasland 2017

Jan Rinze van der Schoot, Henk Schilder 



\section{Rassenbericht Grasland 2017}

Jan Rinze van der Schoot, Henk Schilder

Het Cultuur- en Gebruikswaarde Onderzoek voedergrassen is uitgevoerd door Wageningen Livestock Research in opdracht van en gefinancierd door Plantum.

Wageningen Livestock Research

Wageningen, november 2017

Rapport 1060 
Schoot, J.R. van der, Schilder, H. Rassenbericht Grasland 2017; Wageningen Livestock Research, Rapport 1060.

Samenvatting NL

Rapportage van proefveldresultaten van het rassenonderzoek aan Engels raaigras in de periode 20092016.

Dit rapport is gratis te downloaden op https://doi.org/10.18174/427019 of op www.wur.nl/livestock-research (onder Wageningen Livestock Research publicaties).

(C) 2017 Wageningen UR Livestock Research

Postbus 338, 6700 AH Wageningen, T 03174839 53, E info.livestockresearch@wur.nl, www. wur. nl/livestock-research. Wageningen Livestock Research is onderdeel van Wageningen University \& Research.

Wageningen Livestock Research aanvaardt geen aansprakelijkheid voor eventuele schade voortvloeiend uit het gebruik van de resultaten van dit onderzoek of de toepassing van de adviezen.

Alle rechten voorbehouden. Niets uit deze uitgave mag worden vermenigvuldigd en/of openbaar gemaakt worden door middel van druk, fotokopie, microfilm of op welke wijze dan ook zonder voorafgaande toestemming van de uitgever of auteur.

De certificering volgens ISO 9001 door DNV onderstreept ons kwaliteitsniveau. Op als onze onderzoeksopdrachten zijn de Algemene Voorwaarden van de Animal Sciences Group van D四 toepassing. Deze zijn gedeponeerd bij de Arrondissementsrechtbank Zwolle.

Wageningen Livestock Research Rapport 1060 


\section{Inleiding}

Dit Rassenbericht Grasland 2017 bevat de nieuwste resultaten van het Cultuur- en Gebruikswaarde Onderzoek (CGO) van Engels raaigras. In het CGO worden rassen van voedergrassen en witte klaver getest op hun landbouwkundige waarde voor de veehouder. Bij goede resultaten komen de rassen op de Aanbevelende Rassenlijst. Voor grasland zijn vooral de eigenschappen standvastigheid, ziekteresistentie (zoals resistentie tegen kroonroest bij Engels raaigras), wintervastheid en drogestofopbrengst belangrijk. De eigenschappen worden beoordeeld op beweidings- en maaiproefvelden, waar de nieuwe rassen samen met de bestaande rassenlijstrassen worden uitgezaaid en onderzocht. 


\section{Resultaten}

Weergegeven zijn de proefveldresultaten van het CGO voor Engels raaigras laat en middentijds doorschietend van de afgelopen jaren. Voor de meeste eigenschappen en de opbrengsten hebben de gegevens betrekking op het gemiddelde van de resultaten verkregen in de periode 2009 t/m 2016 . Vermeld zijn de rassenlijstrassen van de Aanbevelende Rassenlijst Veehouderij 2018 en de rassen in onderzoek van de uitzaaijaren 2013 en 2014. De rassen van 2013 zijn reeds beoordeeld voor opname op Rassenlijst 2018.

In dit Rassenbericht Grasland 2017 zijn van Engels raaigras de nieuwe, aanbevolen rassen van Rassenlijst 2018 vermeld met hun waarderingscijfers. De opname en volgorde is gebaseerd op het advies en het persbericht van de Commissie Samenstelling Aanbevelende Rassenlijst (CSAR).

Voor opname is ook inschrijving in het Nederlands Rassenregister en/of EU-verkeerstoelating nodig. De rassen van 2014 worden volgend jaar beoordeeld, omdat het onderzoek nog niet is afgerond. Voor de overige (gras)soorten die in de Aanbevelende Rassenlijst staan vermeld, zijn geen nieuwe gegevens verkregen. Voor meer informatie over grassoorten, graslandmengsels en raseigenschappen wordt verwezen naar de Aanbevelende Rassenlijst Veehouderij 2018. Ook de eigenschappen van de rassenlijstrassen van de andere grassoorten en typen (Engels raaigras vroeg doorschietend, gekruist raaigras, Italiaans raaigras, timothee en beemdlangbloem) en van witte klaver staan hierin vermeld. 


\section{Toelichting op de tabellen}

Het jaartal achter de rasnaam geeft het eerste jaar van uitzaai en onderzoek aan.

( ) betekent: gegevens zijn gebaseerd op weinig waarnemingen.

- betekent: geen of onvoldoende waarnemingen.

Een hoog cijfer betekent een gunstige waardering van de betrokken eigenschap.

De drogestofopbrengst is per soort of type weergegeven in verhoudingsgetallen. Deze zijn gebaseerd op het gemiddelde van de A- en $\mathrm{N}$-rassen van de desbetreffende soort of het type van de Aanbevelende Rassenlijst 2017. Naast de gemiddelde totale jaaropbrengst van de beweidingsproeven en de maaiproeven is ook de opbrengst van de eerste en tweede snede vermeld. $\mathrm{Bij} 100=\ldots$ ton $\mathrm{ds} /$ ha is het opbrengstniveau van de rassenproeven vermeld. De opbrengstniveau's van de beweidingsproeven en de maaiproeven zijn niet direct vergelijkbaar door verschillen in beproevingslocaties en management.

De waarderingscijfers en opbrengstgegevens zijn alleen binnen de soort of het type direct vergelijkbaar. Door verschillen in beproevingsperiode, -omvang en -methode kunnen gegevens niet zondermeer gebruikt worden voor een directe onderlinge vergelijking van typen en soorten.

Afkortingen van namen van kwekers of houders/aanvragers kwekersrecht.

AFBI : Agri-Food and Biosciences Institute, Loughgall, Noord I erland

Bar : Barenbrug Holland B.V., Nijmegen

Car : Carneau Frères S.A., Orchies, Frankrijk

DLF : DLF A/S, Roskilde, Denemarken

DSV : DSV AG, Lippstadt, Duitsland

IBER : Inst. of Biological Environmental \& Rural Sciences, Aberystwyth, Groot Brittannië

ILVO : ILVO - Plant, Melle, België

NPZ : Norddeutsche Pflanzenzucht Hans-Georg Lembke KG, Holtsee, Duitsland

RAGT : RAGT 2n S.A.S., Rodez, Frankrijk

Afkortingen van namen van vertegenwoordigers.
Bar : Barenbrug Holland B.V., Nijmegen
DLF : DLF B.V., Kapelle
DSV : DSV zaden Nederland B.V., Gennep
Joo : Joordens Zaden B.V., Kessel
Lim : Limagrain Nederland B.V., Rilland
VDS : VANDINTER SEMO BV, Scheemda 


\section{ENGELS RAAI GRAS LAAT DOORSCHI ETEND}

Overzicht van de raseigenschappen

\begin{tabular}{|c|c|c|c|c|c|c|c|c|c|}
\hline & \multirow{3}{*}{ 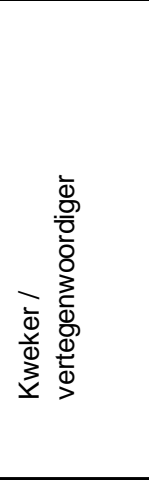 } & \multirow[b]{2}{*}{ 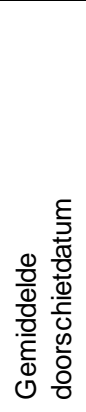 } & \multirow[b]{2}{*}{$\begin{array}{l}\frac{.0}{0} \\
\frac{0}{0} \\
\frac{0}{0} \\
0 \\
\frac{0}{0} \\
0 \\
\frac{\pi}{0} \\
0\end{array}$} & \multirow[b]{2}{*}{ 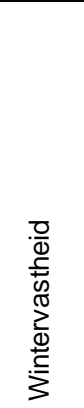 } & \multirow[b]{2}{*}{ 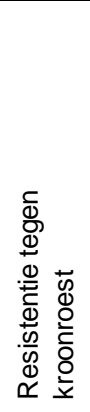 } & \multicolumn{4}{|c|}{$\begin{array}{l}\text { Drogestofopbrengst in } \\
\text { verhoudingsgetallen }\end{array}$} \\
\hline & & & & & & 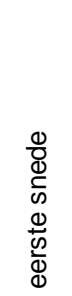 & $\begin{array}{l}\frac{0}{0} \\
\frac{d}{\omega} \\
0 \\
\frac{d}{d} \\
\Phi \\
\vdots\end{array}$ & 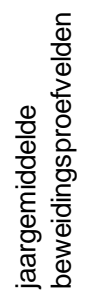 & 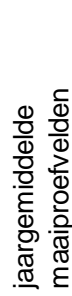 \\
\hline & & 1 & 2 & 3 & 4 & 5 & 6 & 7 & 8 \\
\hline \multicolumn{10}{|l|}{ Rassenlijstrassen } \\
\hline Barnewton & Bar & $4-6$ & 8.4 & 7.6 & 7.8 & 98 & 104 & 103 & 101 \\
\hline Manchester & Bar & $3-6$ & 8.3 & 7.5 & 8.4 & 98 & 101 & 101 & 101 \\
\hline Barimero & Bar & $4-6$ & 8.4 & 7.3 & 7.5 & 100 & 104 & 101 & 100 \\
\hline Rossera & DSV & $2-6$ & 8.0 & 6.9 & 7.8 & 99 & 104 & 102 & 100 \\
\hline Bovini & DLF & $3-6$ & 8.3 & 7.1 & 7.7 & 101 & 96 & 99 & 101 \\
\hline Thomas & Bar & $5-6$ & 8.4 & 6.9 & 7.6 & 103 & 90 & 99 & 99 \\
\hline Humbi 1 & DLF & $4-6$ & 8.2 & 7.4 & 8.1 & 96 & 94 & 100 & 97 \\
\hline Candore & Car/Lim & $2-6$ & 8.4 & 7.2 & 8.0 & 96 & 95 & 98 & 99 \\
\hline Bartombo & Bar & $9-6$ & 8.3 & 7.2 & 7.4 & 97 & 103 & 98 & 100 \\
\hline Romark & DLF & $1-6$ & 8.0 & 7.1 & 7.3 & 98 & 97 & 99 & 98 \\
\hline Melluck & ILVO/Bar & $3-6$ & 8.5 & 6.9 & 7.0 & 98 & 96 & 99 & 97 \\
\hline Tomaso & DSV & $1-6$ & 8.2 & 7.2 & 7.3 & 100 & 96 & 99 & 96 \\
\hline Zenital & RAGT/Joo & $2-6$ & 8.2 & 7.1 & 7.8 & 98 & 99 & 97 & 96 \\
\hline Burlina 1 & DLF & $1-6$ & 8.0 & 6.9 & 7.1 & 97 & 94 & 98 & 99 \\
\hline Jalinas & DLF & $1-6$ & 8.2 & 6.9 & 7.1 & 101 & 94 & 98 & 98 \\
\hline \multicolumn{10}{|l|}{ Nieuwe rassen } \\
\hline Melonora & ILVO/Bar & $4-6$ & 8.5 & 7.0 & 8.3 & 99 & 103 & 101 & 102 \\
\hline Barhoney & Bar & $7-6$ & 8.2 & 7.3 & 8.2 & 97 & 105 & 103 & 102 \\
\hline Mahony & DLF/Lim & $5-6$ & 8.1 & 6.9 & 8.1 & 96 & 105 & 103 & 101 \\
\hline Maiko & DSV & $2-6$ & 8.4 & 7.4 & 7.9 & 97 & 105 & 102 & 101 \\
\hline Tottenham & Bar & $4-6$ & 8.4 & 7.3 & 8.1 & 95 & 102 & 101 & 101 \\
\hline Rossimonte & DSV & $8-6$ & 8.3 & (7.5) & 7.9 & 98 & 109 & 102 & 103 \\
\hline Barbitol & Bar & $3-6$ & 8.2 & (7.5) & 8.2 & 100 & 100 & 102 & 100 \\
\hline \multicolumn{10}{|l|}{ Tetraploïde rassen } \\
\hline Dromara & AFBI/Bar & $2-6$ & 8.1 & 7.6 & 8.9 & 104 & 98 & 99 & 101 \\
\hline Meracoli & ILVO/Bar & $3-6$ & 8.1 & 7.4 & 8.2 & 103 & 98 & 99 & 101 \\
\hline Rivaldo & DSV & $2-6$ & 8.1 & 7.6 & 8.6 & 100 & 100 & 99 & 99 \\
\hline Alcander & DLF/Lim & $2-6$ & 8.1 & 7.5 & 8.8 & 104 & 95 & 97 & 99 \\
\hline Estrada & DSV & $4-6$ & 8.1 & 7.4 & 8.4 & 99 & 103 & 99 & 99 \\
\hline Barpasto & Bar & $2-6$ & 8.1 & 7.9 & 8.0 & 103 & 101 & 98 & 101 \\
\hline Meltador & ILVO/Bar & $5-6$ & 7.8 & 7.5 & 8.0 & 105 & 95 & 98 & 99 \\
\hline Polim & DLF & $1-6$ & 7.6 & 7.5 & 8.1 & 106 & 92 & 96 & 98 \\
\hline Bocage & Car/DLF & $3-6$ & 8.0 & 7.6 & 8.7 & 98 & 94 & 93 & 96 \\
\hline \multicolumn{10}{|l|}{ Nieuwe rassen } \\
\hline Melbolt & ILVO/DLF & $2-6$ & 8.0 & 7.0 & 8.6 & 102 & 103 & 103 & 102 \\
\hline Melkana & ILVO/Bar & $2-6$ & 8.2 & (6.9) & 8.7 & 105 & 105 & 101 & 102 \\
\hline \multicolumn{10}{|c|}{ Nieuwin Rassenlijst 2018} \\
\hline Barganza & Bar & $2-6$ & 7.8 & - & 8.6 & 105 & 103 & 106 & 101 \\
\hline Thegn & DLF & $2-6$ & 7.9 & - & 8.7 & 105 & 104 & 104 & 102 \\
\hline $100=\ldots$ ton ds $/ \mathrm{ha}$ & & & & & & 4.1 & 2.5 & 12.1 & 14.0 \\
\hline
\end{tabular}




\section{Vervolg ENGELS RAAI GRAS LAAT DOORSCHI ETEND}

Overzicht van de raseigenschappen

\begin{tabular}{|c|c|c|c|c|c|c|c|c|c|c|}
\hline & \multirow{3}{*}{ 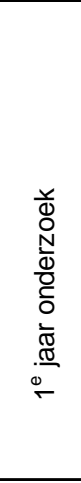 } & \multirow{3}{*}{ 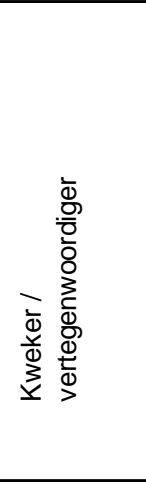 } & \multirow{3}{*}{ 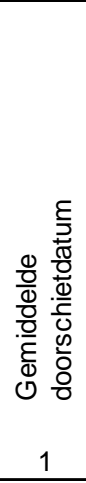 } & \multirow{3}{*}{ 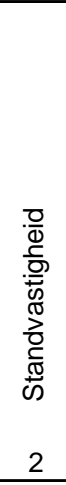 } & \multirow{3}{*}{ 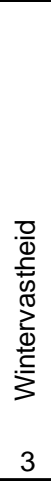 } & \multirow[b]{2}{*}{ 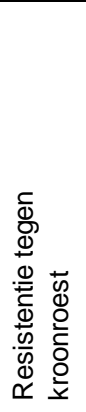 } & \multicolumn{4}{|c|}{$\begin{array}{l}\text { Drogestofopbrengst in } \\
\text { verhoudingsgetallen }\end{array}$} \\
\hline & & & & & & & 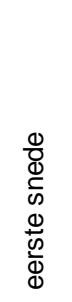 & 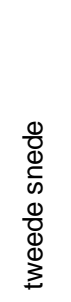 & 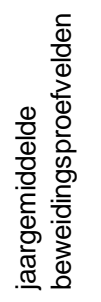 & 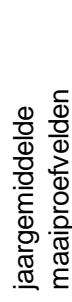 \\
\hline & & & & & & 4 & 5 & 6 & 7 & 8 \\
\hline \multicolumn{11}{|l|}{$\begin{array}{l}\text { Rassen in onderzoek } \\
\text { Diploïde rassen }\end{array}$} \\
\hline Caminero (ELp 060582) & 2013 & DSV & $5-6$ & 8.3 & & 8.1 & 96 & 95 & 97 & 97 \\
\hline 13LPD 132 & 2013 & Bar & $9-6$ & 8.1 & & 7.3 & 88 & 108 & 98 & 98 \\
\hline 13LPD 138 & 2013 & Bar & $4-6$ & 8.2 & & 7.7 & 100 & 92 & 96 & 94 \\
\hline ILVO 135490 & 2013 & ILVO/Bar & $4-6$ & 8.4 & & 8.0 & 95 & 102 & 97 & 99 \\
\hline ILVO 135773 & 2013 & ILVO/Bar & $1-6$ & 8.2 & & 8.1 & 100 & 93 & 100 & 98 \\
\hline Jasmund (SLM22301/07) & 2013 & NPZNDS & $3-6$ & 8.2 & & 7.5 & 102 & 95 & 99 & 98 \\
\hline Valmiron (LMG LFD-52764) & 2013 & DLF/Lim & $1-6$ & 8.1 & & 7.7 & 100 & 99 & 101 & 100 \\
\hline DLF LFD-42477 & 2013 & DLF & $29-5$ & 8.0 & & 7.9 & 101 & 92 & 98 & 98 \\
\hline Maredo (Elp 062130) & 2014 & DSV & $4-6$ & 8.1 & & 7.8 & 93 & 99 & 99 & 97 \\
\hline Andena (Elp 210481) & 2014 & DSV & $31-5$ & 8.0 & & 7.7 & 108 & 95 & 103 & 99 \\
\hline 14LPD 113 & 2014 & Bar & $4-6$ & 8.2 & & 7.7 & 92 & 108 & 96 & 101 \\
\hline 14LPD 118 & 2014 & Bar & $5-6$ & 7.9 & & 8.0 & 95 & 111 & 106 & 101 \\
\hline 14LPD 123 & 2014 & Bar & $5-6$ & 7.7 & & 7.5 & 91 & 109 & 102 & 98 \\
\hline RGAS974 & 2014 & RAGT/Joo & $1-6$ & 8.4 & & 8.4 & 98 & 96 & 99 & 98 \\
\hline DT 252 & 2014 & Car/Lim & $4-6$ & 8.2 & & 8.5 & 97 & 93 & 100 & 98 \\
\hline DLF LFD-62760 & 2014 & DLF & $2-6$ & 8.1 & & 7.8 & 91 & 97 & 96 & 91 \\
\hline DLF LFD-21352 & 2014 & DLF & $26-5$ & 8.2 & & 7.9 & 101 & 93 & 99 & 97 \\
\hline DLF LFD-21385 & 2014 & DLF & $2-6$ & 8.4 & & 8.0 & 93 & 100 & 100 & 92 \\
\hline \multicolumn{11}{|l|}{ Tetraploïde rassen } \\
\hline Rolinde (ELp 04-713) & 2013 & DSV & $2-6$ & 8.0 & & 8.7 & 101 & 101 & 99 & 98 \\
\hline 13LPT 214 & 2013 & Bar & $6-6$ & 8.1 & & 8.5 & 97 & 92 & 95 & 96 \\
\hline $\mathrm{TI} 47$ & 2013 & Carn/Lim & $2-6$ & 8.2 & & 8.7 & 110 & 89 & 99 & 101 \\
\hline DLF LFT-42487 & 2013 & DLF & $31-5$ & 7.6 & & 7.0 & 105 & 95 & 96 & 98 \\
\hline Kassian (LMG LFT-4009) & 2013 & DLF/Lim & $2-6$ & 8.0 & & 8.4 & 103 & 97 & 99 & 100 \\
\hline 13LPT 3083 & 2014 & Bar & $3-6$ & 7.9 & & 8.7 & 100 & 96 & 94 & 94 \\
\hline 14LPT 215 & 2014 & Bar & $31-5$ & 7.8 & & 8.8 & 106 & 97 & 101 & 102 \\
\hline 14LPT 224 & 2014 & Bar & $31-5$ & 8.0 & & 8.8 & 108 & 93 & 100 & 101 \\
\hline |LVO 145914 & 2014 & ILVO/Bar & $1-6$ & 8.3 & & 8.9 & 102 & 97 & 98 & 104 \\
\hline Boudica (DLF LFT-41335) & 2014 & DLF & $27-5$ & 7.8 & & 8.5 & 113 & 91 & 99 & 104 \\
\hline Mellara (ILVO142932) & 2014 & ILVO/DLF & $4-6$ & 8.3 & & 8.5 & 101 & 104 & 100 & 102 \\
\hline Salvina (LMG LFT-41351) & 2014 & DLF/Lim & $1-6$ & 8.1 & & 8.8 & 107 & 97 & 99 & 104 \\
\hline $100=\ldots$ ton ds $/$ ha & & & & & & & 4.1 & 2.5 & 12.1 & 14.0 \\
\hline
\end{tabular}




\section{ENGELS RAAI GRAS MI DDENTIJ DS}

Overzicht van de raseigenschappen

\begin{tabular}{|c|c|c|c|c|c|c|c|c|c|}
\hline & \multirow{3}{*}{ 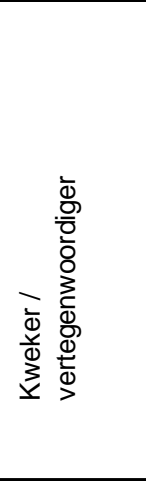 } & \multirow[b]{2}{*}{ 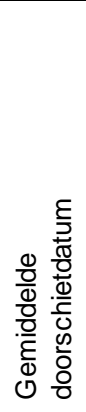 } & \multirow[b]{2}{*}{ 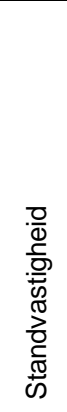 } & \multirow[b]{2}{*}{ 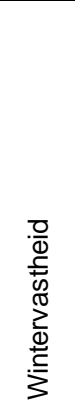 } & \multirow[b]{2}{*}{ 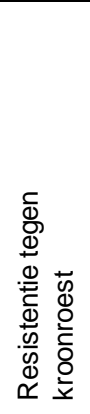 } & \multicolumn{4}{|c|}{$\begin{array}{l}\text { Drogestofopbrengst in } \\
\text { verhoudingsgetallen }\end{array}$} \\
\hline & & & & & & $\begin{array}{l}\frac{0}{0} \\
\frac{d}{n} \\
0 \\
\Phi \\
\frac{0}{0} \\
\frac{0}{0}\end{array}$ & 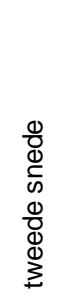 & 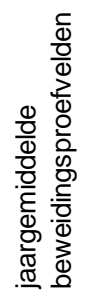 & 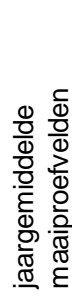 \\
\hline & & 1 & 2 & 3 & 4 & 5 & 6 & 7 & 8 \\
\hline \multicolumn{10}{|l|}{ Rassenlijstrassen } \\
\hline Sputnik & DLF & $29-5$ & 8.2 & 6.5 & 8.4 & 104 & 103 & 108 & 103 \\
\hline Arnando & DSV & $29-5$ & 8.5 & 7.5 & 8.0 & 95 & 108 & 102 & 100 \\
\hline Arsenal & DSV & $22-5$ & 8.2 & 7.1 & 7.6 & 104 & 92 & 100 & 101 \\
\hline Abosan 1 & DLF & $29-5$ & 8.2 & 7.0 & 8.1 & 96 & 100 & 101 & 100 \\
\hline Kiani & DLF & $31-5$ & 8.3 & 7.5 & 8.3 & 97 & 102 & 101 & 96 \\
\hline Trenio & DSV & $22-5$ & 8.4 & 6.7 & 7.9 & 98 & 101 & 99 & 99 \\
\hline Massimo & DLF/Lim & $25-5$ & 8.2 & 6.6 & 7.8 & 97 & 105 & 99 & 101 \\
\hline Kaiman & DSV & $30-5$ & 7.9 & 6.9 & 7.7 & 95 & 110 & 100 & 102 \\
\hline Toronto & DSV & $22-5$ & 8.2 & 6.8 & 7.2 & 105 & 89 & 100 & 98 \\
\hline Chicago & DSV & $23-5$ & 8.7 & 6.9 & 7.4 & 97 & 97 & 97 & 99 \\
\hline Domiatti 1 & DLF & $29-5$ & 7.9 & 6.5 & 7.9 & 97 & 94 & 97 & 98 \\
\hline Option & DLF & $25-5$ & 8.2 & 7.0 & 7.9 & 96 & 96 & 97 & 97 \\
\hline \multicolumn{10}{|l|}{ Nieume rassen } \\
\hline Arelio & DSV & $23-5$ & 8.4 & 7.3 & 7.6 & 105 & 102 & 106 & 102 \\
\hline Melspring & ILVO/Bar & $25-5$ & 8.4 & 6.8 & 8.3 & 103 & 89 & 101 & 101 \\
\hline \multicolumn{10}{|c|}{ Tetraploïde rassen } \\
\hline Diwan & DLF & $27-5$ & 7.9 & 7.6 & 8.7 & 105 & 99 & 101 & 102 \\
\hline Binario & DSV & $22-5$ & 7.9 & 7.7 & 8.8 & 103 & 93 & 99 & 100 \\
\hline Barcampo & Bar & $28-5$ & 8.1 & 7.8 & 8.9 & 99 & 102 & 99 & 100 \\
\hline Barfamos & Bar & $28-5$ & 8.1 & 7.8 & 8.6 & 96 & 112 & 100 & 100 \\
\hline Trivos & DSV & $26-5$ & 7.8 & 7.1 & 8.6 & 101 & 103 & 97 & 101 \\
\hline Maurizio & DSV & $22-5$ & 8.1 & 7.7 & 8.4 & 104 & 98 & 97 & 100 \\
\hline Hurricane & Car/Lim & $29-5$ & 8.0 & 7.2 & 8.7 & 98 & 106 & 98 & 98 \\
\hline Activa & Car/VDS & $21-5$ & 7.8 & 7.5 & 8.5 & 103 & 90 & 97 & 97 \\
\hline Novello & DLF/Lim & $31-5$ & 8.1 & 7.3 & 8.3 & 90 & 117 & 98 & 101 \\
\hline Melverde & ILVO/Bar & $24-5$ & 8.1 & 7.0 & 8.5 & 96 & 102 & 97 & 99 \\
\hline Besser & DLF & $25-5$ & 7.8 & 7.5 & 8.0 & 100 & 107 & 97 & 100 \\
\hline \multicolumn{10}{|l|}{ Nieuwe rassen } \\
\hline Briant & Bar & $30-5$ & 7.9 & (7.1) & 9.0 & 99 & 108 & 101 & 102 \\
\hline Vifelt & DLF & $24-5$ & 8.0 & $(7.5)$ & 8.7 & 99 & 98 & 100 & 98 \\
\hline \multicolumn{10}{|c|}{ Nieuw in Rassenlijst 2017} \\
\hline Barojet & Bar & $27-5$ & 8.0 & - & 8.7 & 100 & 111 & 100 & 107 \\
\hline AberGain & IBER/VDS & $28-5$ & 7.8 & - & 8.5 & 109 & 107 & 99 & 106 \\
\hline Velonit & DLF & $24-5$ & 7.9 & - & 8.8 & 106 & 98 & 101 & 101 \\
\hline Magena & ILVO/DLF & $31-5$ & 8.2 & - & 8.6 & 95 & 118 & 101 & 101 \\
\hline $100=\ldots$ ton $d s / h a$ & & & & & & 4.7 & 2.1 & 12.3 & 14.3 \\
\hline
\end{tabular}




\section{Vervolg ENGELS RAAI GRAS MI DDENTI J DS}

Overzicht van de raseigenschappen

\begin{tabular}{|c|c|c|c|c|c|c|c|c|c|c|}
\hline & \multirow{3}{*}{ 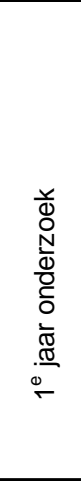 } & \multirow{3}{*}{ 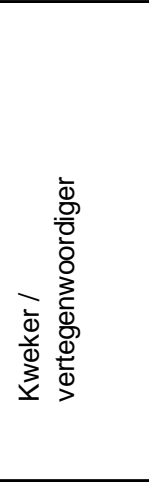 } & \multirow{3}{*}{ 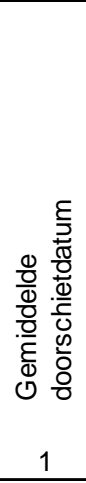 } & \multirow{3}{*}{ 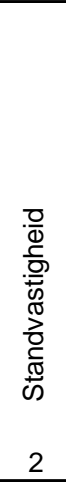 } & \multirow{3}{*}{ 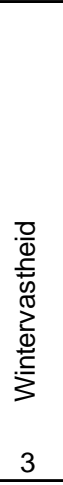 } & \multirow[b]{2}{*}{ 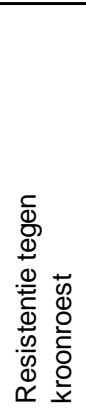 } & \multicolumn{4}{|c|}{$\begin{array}{l}\text { Drogestofopbrengst in } \\
\text { verhoudingsgetallen }\end{array}$} \\
\hline & & & & & & & $\begin{array}{l}\frac{d}{0} \\
\frac{d}{\omega} \\
\omega \\
\Phi \\
\frac{\omega}{0} \\
\Phi\end{array}$ & 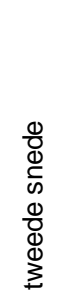 & 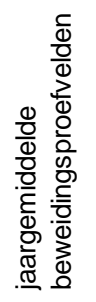 & 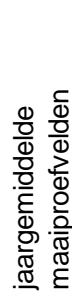 \\
\hline & & & & & & 4 & 5 & 6 & 7 & 8 \\
\hline \multicolumn{11}{|l|}{$\begin{array}{l}\text { Rassen in onderzoek } \\
\text { Diplö̈de rassen }\end{array}$} \\
\hline Lanzaco (ELp 04-604) & 2013 & DSV & $27-5$ & 8.2 & - & 8.1 & 92 & 103 & 94 & 99 \\
\hline 13LPD 108 & 2013 & Bar & $27-5$ & 8.2 & - & 7.1 & 89 & 94 & 91 & 94 \\
\hline 13LPD 117 & 2013 & Bar & $29-5$ & 8.1 & - & 8.4 & 92 & 105 & 97 & 99 \\
\hline 13LPD 3055 & 2013 & Bar & $25-5$ & 8.1 & - & 8.1 & 101 & 88 & 97 & 99 \\
\hline DLF LFD-7030 & 2013 & DLF & $17-5$ & 8.2 & - & 7.7 & 110 & 94 & 102 & 105 \\
\hline Wisram (DLF LFD-8511) & 2013 & DLF & $23-5$ & 8.1 & - & 8.1 & 101 & 92 & 101 & 101 \\
\hline DLF LFD-21496 & 2013 & DLF & $28-5$ & 7.4 & - & 7.2 & 87 & 102 & 88 & 93 \\
\hline Armetto (ELp 03-629) & 2014 & DSV & $22-5$ & 8.1 & - & 8.5 & 104 & 95 & 102 & 101 \\
\hline RGAS971 & 2014 & RAGT/Joo & $27-5$ & 8.2 & - & 8.7 & 92 & 98 & 99 & 97 \\
\hline DLF LFD-62637 & 2014 & DLF & $26-5$ & 8.1 & - & 7.8 & 94 & 98 & 95 & 93 \\
\hline DLF LFD-21397 & 2014 & DLF & $22-5$ & 8.0 & - & 7.4 & 99 & 93 & 96 & 98 \\
\hline Maqui (DLF LFD-21422) & 2014 & DLF & $26-5$ & 8.2 & - & 8.4 & 99 & 96 & 103 & 99 \\
\hline Verdasco (LMG LFD-2061) & 2014 & DLF/Lim & $22-5$ & 7.7 & - & 7.8 & 107 & 91 & 101 & 98 \\
\hline Aberwolf (Ba14074) & 2014 & IBER/VDS & $24-5$ & 8.3 & - & 7.6 & 99 & 97 & 99 & 99 \\
\hline \multicolumn{11}{|l|}{ Tetraploïde rassen } \\
\hline Aberclyde (Ba13940) & 2013 & IBER/VDS & $19-5$ & 7.6 & - & 8.6 & 104 & 93 & 93 & 99 \\
\hline Marniere (LMG LFT-41317) & 2013 & DLF/Lim & $26-5$ & 7.8 & - & 8.7 & 98 & 104 & 98 & 100 \\
\hline DLF LFT-44461 & 2013 & DLF & $26-5$ & 7.1 & - & 8.5 & 99 & 101 & 93 & 97 \\
\hline DLF LFT-74626 & 2013 & DLF & $29-5$ & 7.0 & - & 8.6 & 95 & 102 & 94 & 94 \\
\hline Arapho (DLF LFT-74925) & 2013 & DLF & $26-5$ & 7.9 & - & 8.5 & 102 & 99 & 97 & 97 \\
\hline Explosion (ELp 04-689) & 2014 & DSV & $22-5$ & 8.0 & - & 8.8 & 115 & 95 & 107 & 107 \\
\hline 14LPT 205 & 2014 & Bar & $26-5$ & 8.0 & - & 9.0 & 97 & 105 & 97 & 102 \\
\hline 14LPT 210 & 2014 & Bar & $30-5$ & 8.2 & - & 8.9 & 93 & 110 & 103 & 102 \\
\hline 14LPT 213 & 2014 & Bar & $31-5$ & 8.0 & - & 8.9 & 100 & 107 & 104 & 101 \\
\hline 14LPT 223 & 2014 & Bar & $29-5$ & 8.1 & - & 8.8 & 96 & 113 & 103 & 102 \\
\hline Matenga (SLM 24233/04) & 2014 & NPZNDS & $24-5$ & 8.0 & - & 8.3 & 109 & 93 & 100 & 101 \\
\hline Quadriga (SLM 24215/03) & 2014 & NPZIVDS & $29-5$ & 8.1 & - & 6.9 & 95 & 105 & 98 & 96 \\
\hline TRAH962 & 2014 & RAGT/Joo & $26-5$ & 8.1 & - & 8.9 & 103 & 96 & 97 & 100 \\
\hline DLF LFT-0808B2 & 2014 & DLF & $21-5$ & 4.0 & - & 8.5 & 102 & 88 & 80 & 86 \\
\hline ILVO145903 & 2014 & ILVO/DLF & $28-5$ & 8.2 & - & 9.1 & 103 & 107 & 108 & 107 \\
\hline Weldone (LMG LFT-41349) & 2014 & DLF/Lim & $28-5$ & 8.2 & - & 8.9 & 95 & 115 & 102 & 105 \\
\hline $100=\ldots$ ton ds $/ \mathrm{ha}$ & & & & & & & 4.7 & 2.1 & 12.3 & 14.3 \\
\hline
\end{tabular}





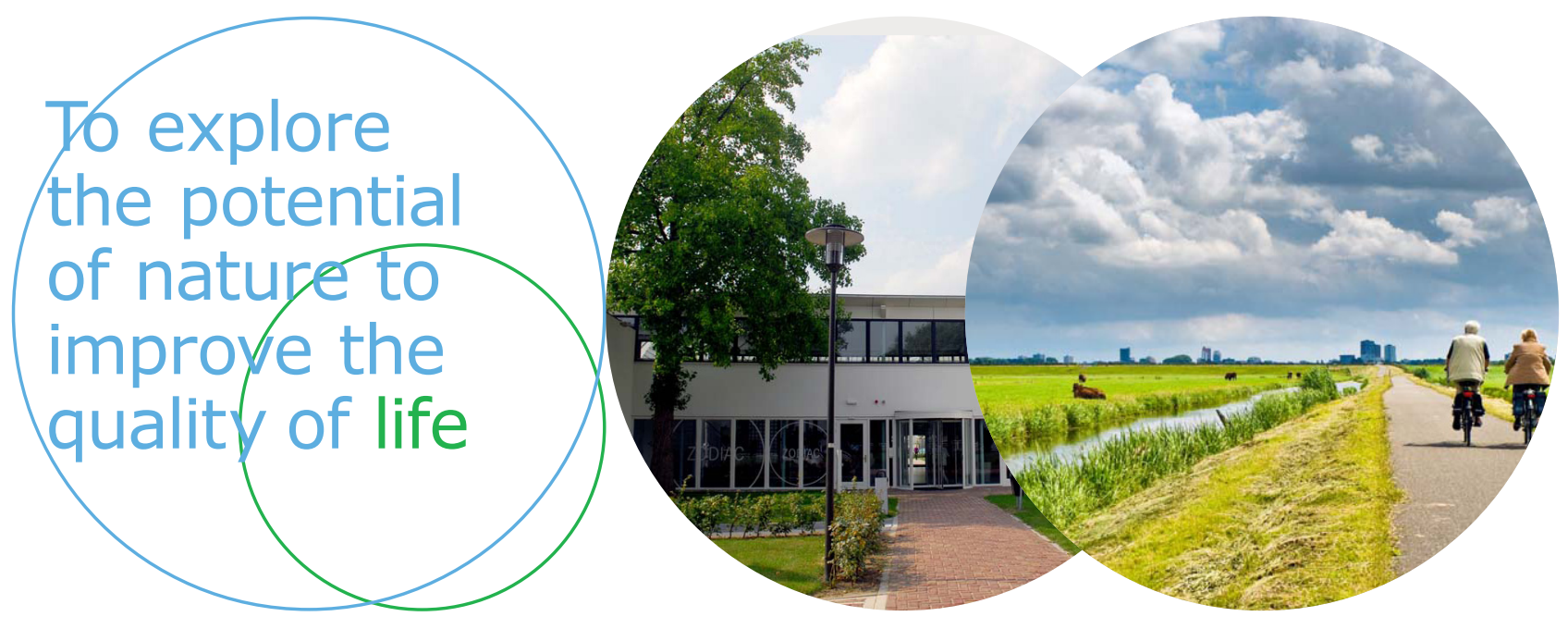

Wageningen Livestock Research Postbus 338

Wageningen Livestock Research ontwikkelt kennis voor een zorgvuldige en $6700 \mathrm{AH}$ Wageningen

T 0317483953

renderende veehouderij, vertaalt deze naar praktijkgerichte oplossingen en innovaties, en zorgt voor doorstroming van deze kennis. Onze wetenschappelijke E info.livestockresearch@wur.nl www.wur.nl/ livestock-research kennis op het gebied van veehouderijsystemen en van voeding, genetica, welzijn en milieu-impact van landbouwhuisdieren integreren we, samen met onze klanten, tot veehouderijconcepten voor de $21 \mathrm{e}$ eeuw.

De missie van Wageningen University \& Research is 'To explore the potential of nature to improve the quality of life'. Binnen Wageningen University \& Research bundelen 9 gespecialiseerde onderzoeksinstituten van Stichting Wageningen Research en Wageningen University hun krachten om bij te dragen aan de oplossing van belangrijke vragen in het domein van gezonde voeding en leefomgeving. Met ongeveer 30 vestigingen, 6.500 medewerkers en 10.000 studenten behoort Wageningen University \& Research wereldwijd tot de aansprekende kennisinstellingen binnen haar domein. De integrale benadering van de vraagstukken en de samenwerking tussen verschillende disciplines vormen het hart van de unieke Wageningen aanpak. 